\title{
LE dÉVELOPPEMENT DE LA SÉcrétION LACTÉE (1)
}

\author{
par
}

\author{
JOHN HAMMOND \\ Ecole d'Agriculture, Université de Cambridge. (2)
}

Les premiers travaux de Ch. Porcher sur la formation du lactose dans la glande mammaire [7] ont donné lieu à de nombreuses recherches ayant en vue la détermination du mode exact de la formation du lait dans la mamelle. Le récent travail [8] de cet auteur nous a présenté le lait sous un aspect qui nous a permis de reviser nos connaissances sur le processus de la formation du lait et de son utilisation.

- Puisque le développement chez l'individu présente les mêmes aspects successifs que ceux que nous offre l'évolution de l'animal, l'étude du début du processus de la lactation ehez l'individu devrait nous montrer comment a lieu l'évolution de la mamelle et nous fournir l'explication des différences qui existent entre les laits des différentes espèces animales.

Les modifications qui se produisent pendant le début de la lactation chez l'individu seront examinées à trois points de vue :

a) Morphologie ;

b) Quantité de produit sécrété ;

c) Composition chimique des sécrétions.

a) Morphologie. - La formation des glandes mammaires débute par un épaississement suivi d'une prolifération de l'épiderme cutané : celui-ci s'évide pour former la eiterne lactifère de laquelle irradient un grand nombre de canaux galactophores primaires. La mamelle de la génisse vierge est constituée par un grand nombre de ces canaux comprenant de petits canaux lobulaires formés à l'époque de la puberté : pendant les 20 premières semaines de la gestation, on n'y observe que peu de modifications. A ce moment ( $5^{\mathrm{e}}$ mois de la gestation), les canaux commencent à bourgeonner et à former des alvéoles, lesquelles seront tapissées par les cellules sécrétrices du lait; ces canaux augmentent en nombre et en dimension jusqu'à la fin de la gestation [4]. Jusqu'à environ la moitié de la première gestation, les cellules de la glande mammaire conservent les fonctions des cellules de l'épiderme cutané, et nous en trouvons la preuve chez le pore noir. Chez cet animal, en effet, la couche malpighienne de la peau contient des granulations de mélanine, qu'on rencontre aussi dans les cellules de la mamelle jusqu'à la phase susindiquée de son développement [5]. Toutefois, passé celle-ci, c'est-à-dire à peu près

(1) Paru dans le Volume jubilaire du Professeur Ch. Porcher.

(2) Je remercie vivement M. R. N. Göransson d'avoir bien voulu effectuer la traduction de mon travail en français. (J. H.) 
vers la moitié de la gestation, le métabolisme des cellules alvéolaires se modifie, et elles ne produisent plus de pigment noir ; le métabolisme cutané de la cellule s'est transformé en un métabolisme lacté.

b) Quantité de produit sécrété. - La sécrétion lactée ne débute pas immédiatement après la parturition; c'est une fonction qui se développe graduellement. La courbe de la lactation, dont on ne tient généralement compte qu'à partir de la parturition jusqu'au moment où la vache est "sèche", est constituée par une courbe qui est d'abord ascendante, ensuite descendante. Ceci fut déterminé par AsDELL [1], qui opéra régulièrement la traite entre la conception et la parturition chez une génisse, done chez unefemelle fécondée pour la première fois. Des chiffres obtenus par lui, il a été établi un diagramme (voir graphique) représentant une courbe de lactation complète.

$\mathrm{Si}$ on effectue la traite des mamelons de génisses vierges, on peut souvent obtenir une petite quantité variable de liquide séreux clair. Pour un animal déterminé, la quantité de liquide ainsi obtenue est relativement constante, la sécrétion étant équilibrée par l'absorption ; mais, si on élimine la sécrétion par une traite plus fréquente, on en obtient une plus grande proportion. Cette sécrétion séreuse persiste pendant la première période de la gestation; mais, à partir de la vingtième semaine, soit du cinquième mois, de la gestation, elle augmente graduellement jusqu'au moment de la parturition. Chez les vaches dont l'état de nutrition est très bon, et dont la traite est exécutée trois fois par jour, pour éviter que la pression intra-mammaire empêche la formation de la sécrétion, celle-ci continue à augmenter jusque vers la sixième semaine après la parturition. Elle diminue ensuite régulièrement jusqu'à la fin de la lactation.

La forme exacte de la courbe de lactation varie avec le degré de nutrition, exactement comme une courbe de croissance, ce qu'elle est, du reste, en réalité. L'administration aux vaches, avant vêlage, d'une ration très riche en protéine ayant pour but une forte production de lait (pratique dénommée "steaming up " par les éleveurs anglais), produit un plus grand développement de la glande et une ascension plus rapide de la courbe, qui, non seulement, atteint un niveau plus élevé, ce qui a pour résultat une production plus abondante de lait après le vêlage [3], mais provoque aussi, très fréquemment, une sécrétion lactée d'une abondance telle qu'il est nécessaire de pratiquer la traite avant le vêlage. La forme de la courbe après le vêlage dépend étroitement des conditions de la nutrition (SANDERs [9]). Quand celles-ci sont mauvaises, elle commence à descendre immédiatement après la parturition, sans être montée initialement. Toutefois, si les conditions de la nutrition sont les meilleures, la courbe montera pendant quatre à six semaines. Pour que cette ascension de la courbe ait lieu, il faut que la vache reçoive une ration șupérieure à celle qui 
est nécessaire pour la production du lait, du moment. On n'a pas pu encore déterminer, jusqu'à présent, quel est le facteur déterminant

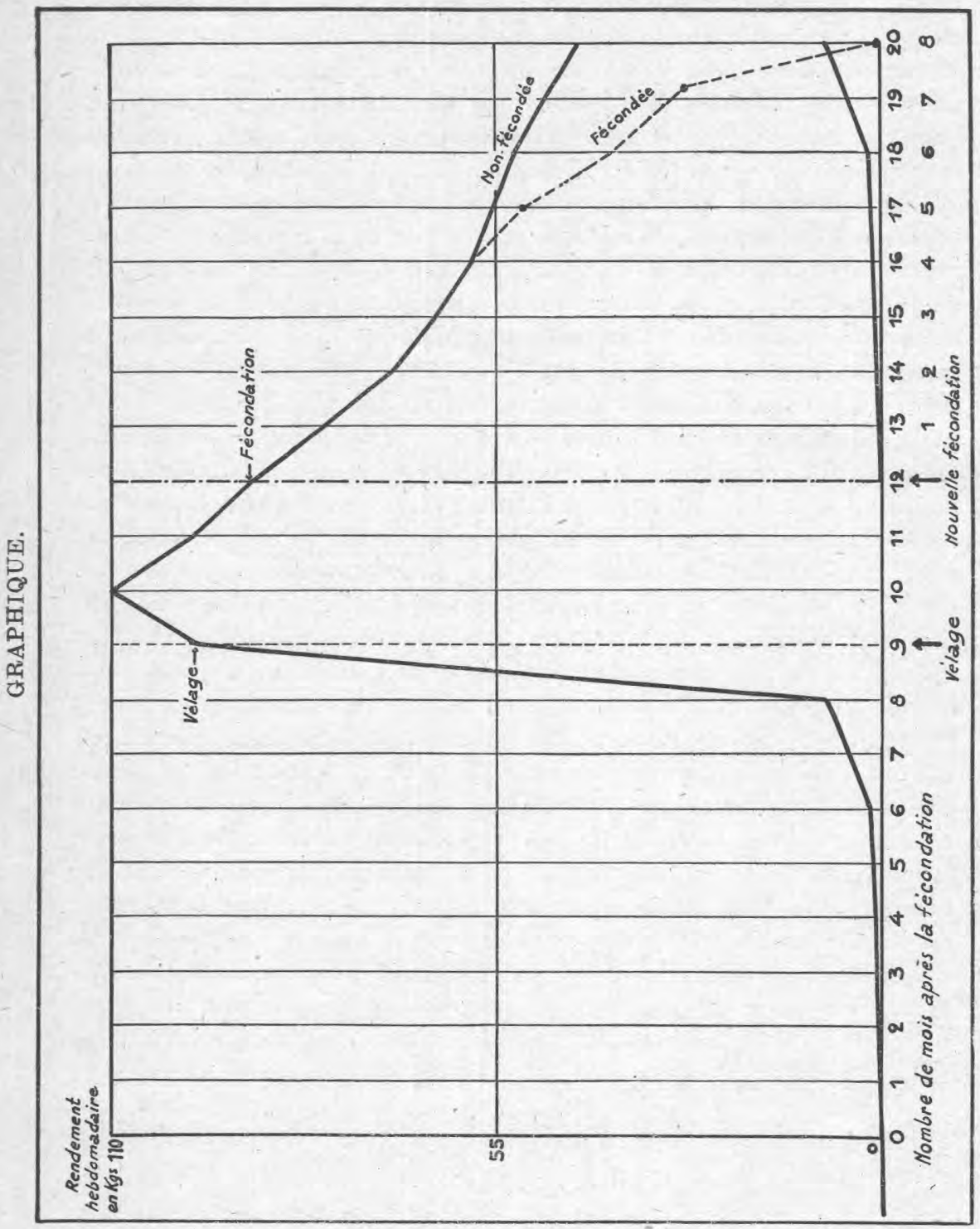

de cette ascension. Celle-ci est-elle due au "stimulant " qui détermine le développement de la mamelle au cours de la gestation, et dont l'action persisterait, ou bien est-elle uniquement attribuable à une intensité plus grande de l'activité sécrétrice, indépendante de 
l'augmentation de l'étendue de la surface glandulaire, qui se produirait pendant les six semaines qui suivent la parturition? Nous sommes enclin à penser que cette augmentation peut être due à la mise en pleine activité de nombreuses cellules déjà formées, mais encore incomplètement développées, lorsque le "stimulant " du développement de la mamelle est éliminé par la parturition, cellules dont l'activité serait faible ou nulle si les conditions de la nutrition étaient mauvaises ; il y a done intensification de l'activité sécrétrice.

Le sommet de la courbe s'élève à chaque lactation jusqu'à la septième [9]. Ceci est probablement dû à ce que la croissanee du tissu mammaire dans chaque lactation nouvelle part d'un niveau plus élevé que lors de la lactation qui précède. Cependant, à la huitième lactation, la sénilité de l'animal entier commence à se manifester et la courbe ne monte pas si haut. Ce début de sénilité se manifeste, même dans les lactations antérieures, par la rapidité plus grande avec laquelle la courbe tombe dès que le maximum a été atteint. Quand, ainsi que cela se produit généralement, les courbes de lactation se succèdent rapidement, le commencement de la seconde courbe ascendante recouvre la partie descendante de la première courbe, et, comme il est impossible à la glande mammaire de sécréter du lait et de se développer simultanément au degré maximum, l'une des deux fonctions, ou les deux à la fois, présente une réduction d'activité. Après la vingtième semaine de gestation, le rendement d'une vache en état de lactation diminue rapidement (voir sur le graphique, le point de la courbe marqué par une ligne pointillée). En outre, si la traite est continuée jusqu'au vêlage, la lactation suivante donnera beaucoup moins de lait, parce que la glande mammaire n'aura pas eu la période de repos nécessaire à son complet développement. Le rendement en lait de la lactation suivante augmente beaucoup, proportionnellement à la durée pendant laquelle la vache est "sèche ", de 1 à 40 jours avant le vêlage ; mais si cette période de " sécheresse " dépasse 60 jours, on n'obtient qu'une très légère augmentation pour les journées en surnombre, ce qui prouve qu'une période de 40 à 60 jours est nécessaire pour que le maximum de développement de la mamelle se produise.

Ce qui prouve que le développement des glandes mammaires, qui se produit pendant la dernière partie de la gestation, est la cause essentielle de la production du lait, plutôt que n'importe quel autre facteur ayant trait à la naissance, c'est que lorsque la gestation, chez la vache, se termine par un avortement avant le einquième mois (ou chez la chèvre à l'époque correspondante, qui est la $10^{\mathrm{e}}$ semaine), il n'y a aucune production de lait, tandis que si l'avortement a lieu plus tard, il y a production de lait, production qui est d'autant plus importante que le temps écoulé depuis l'époque indiquée ci-dessus est plus long [2]. 
c) Composition chimique des produits sécrétés. - Les produits sécrétés par les mamelles des génisses vierges et des génisses pleines contiennent jusqu'à la vingtième semaine de faibles proportions de lactose $(1,2 \%)$ et de matière grasse $(0,1 \%)$, en même temps qu'un peu de caséine $(2,4 \%)$ et d'albumine $(1,6 \%)$; mais beaucoup plus de globuline $(3,4 \%)$ [10]. On peut donc en conclure que la glande qui élabore les constituants normaux du lait ne montre qu'une faible activité.

Cependant, dès que les alvéoles commencent à se développer à la vingtième semaine de gestation, le caractère de la sécrétion se modifie. Les constituants caractéristiques du lajt tendent à disparaître et, en fin de compte, la sécrétion, qui a, à ce moment, la consistance du miel, contient environ $40 \%$ de constituants solides, dont $35 \%$ environ sont des globulines [11]. L'élimination de cette sécrétion de consistance analogue au miel donne lieu à un redoublement de l'activité normale de la glande mammaire, de la même façon que des traites complètes et fréquentes stimulent son activité pendant la période normale de lactation. La sécrétion visqueuse est remplacée par un liquide laiteux qui contient, tout d'abord, des pourcentages appréciables des constituants normaux du colostrum, parmi lesquels il faut citer spécialement la globuline. Celle-ci diminue graduellement au fur et à mesure que la proportion de lait augmente et que l'époque normale de la parturition approche [1]. Si la sécrétion de consistance analogue à celle du miel n'est pas éliminée de la glande, le passage de cette phase à la phase colostrale semble être plus lent, ce qui revient à dire que la pratique de la traite tend à favoriser l'activité sécrétrice et à diminuer l'activité de développement.

Les faits les plus significatifs relatifs à ces changements de la composition pendant le début de la lactation sont les suivants : Quand l'activité de la glande est faible, les proportions de globuline et d'albumine par rapport à celles de la caséine sont élevées, tandis que pendant la période durant laquelle la glande se prépare à une activité plus accentuée, la proportion de globuline est considérablement augmentée. Quand la glande est en pleine activité, la proportion de globuline par rapport à celle de caséine est très faible. On peut, par conséquent, classer les laits des différentes espèces animales en se basant sur le développement de cette évolution. Les laits de quelques espèces (jument, chienne, femme) contiennent encore de fortes proportions de globulines et d'albumines lorsque l'activité sécrétrice est la plus élevée; il est probable qu'ils sont comparables au lait de vache pendant la période de début de la lactation.

La quantité de lait produite par un animal peut être augmentée de deux façons : a) par une augmentation de la surface du tissu glandulaire; b) par une augmentation de l'activité sécrétrice. La 
composition particulière (très riche en globulines) du lait de jument peut être expliquée en supposant que son évolution a tout d'abord eu lieu de la première manière, tandis que, dans le cas de la vache, l'évolution a d'abord eu lieu par l'augmentation de l'activité sécrétrice, ce qui est prouvé par l'augmentation de la proportion de caséine ęt la réduction de celles de la globuline et de l'albumine.

A l'appui de cette hypothèse, on peut, dans une certaine mesure, noter les variations de composition du lait de vache. Au fur et à mesure qu'elle vieillit, jusqu'à la sixième lactation, le rendement augmente, ce qui est dû à l'augmentation de la surface glandulaire : il en résulte que la composition du lait est à peine modifiée. Toutefois, au cours d'une lactation, lorsque le rendement est sujet à des fluctuations liées à l'intensité variable de l'activité sécrétrice, il y a de fortes variations dans la composition du lait : la proportion de easéine dans les constituants solides du lait atteint son maximum à la sixième semaine après la parturition, lorsque le rendement en lait est le plus élevé, tandis que, vers la fin de la courbe de lactation, la proportion de caséine dans les constituants solides du lait diminue, ce qui correspond à une augmentation de la matière grasse, de la globuline et de l'albumine. Il semble probable que l'évolution du lait chez la vache ait lieu par une augmentation de la production d'eau, de lactose et de caséine, celle-ci s'élaborant aux dépens du premier constituant, qui en est peut-être aussi le précurseur, la globuline, la quantiţé produite de cette dernière substance dépendant plus d'une augmentation de la surface glandulaire que d'une exacerbation de l'activité sécrétrice.

La connaissance des variations de la proportion de globuline dans le lait trouve des applications dans plusieurs domaines. Les substances immunisantes dans les sérums préparés pour la prévention contre certaines maladies sont associées à la globuline. Puisque la sécrétion de consistance analogue à celle du miel obtenue au cinquième mois, contient $35 \%$ de globuline, elle doit constituer une source très concentrée d'antitoxines. ORLA-JENSEN [6] a montré que la montée de la crème est due aux propriétés agglutinantes de la globuline. La montée de la crème est très marquée dans le colostrumainsi que le montre la formation des corpuscules de colostrum, (agglutination des globules de matière grasse). Puisque la globuline est coagulée par la pasteurisation, un grand nombre des inconvénients qui résultent de cette opération, tels que, par exemple, la montée lente et peu abondante de la crème (si importante dans le commerce du lait en bouteilles, qui augmente constamment), peuvent être évités par son addition au lait.

Il a été également constaté que des défauts de qualité se produisent fréquemment dans la fabrication du fromage préparé avee du lait 
pasteurisé. Ces défectuosités peuvent être dues à la destruction de la globuline et de l'albumine, car, comme Ch. Porcher [8] l'a montré, la consistance du coagulum produit par la présure est nettement influencée par la présence de protéines telles que l'albumine et la globuline, et ceci à un degré tel, que, dans les laits globulineux (femme, jument, chienne), l'action protectrice de ces substances empêche la formation d'un véritable caillé. La formation d'un caillé consistant, tel que celui qui se produit lorsque la globuline et l'albumine sont absentes, est cause d'un grand nombre de troubles digestifs chez les petits enfants et chez les jeunes animaux alimentés au lait de vache. La connaissance des variations naturelles de la composition des laits a donc, à ce point de vue, des applications importantes dans l'alimentation de tous les jeunes animaux.

(Traduction de R. N. Göransson.)

\title{
BIBLIOGRAPHIE.
}

[1] Asdell. Journ. Agr. Sc., 1925, 15.

[2] Asdell et Robinson. Journ. Physiol., 1926, 61.

[3] Boutflour. World's Dairy Congress, Londres, 1928. Proceedings.

[4] Hammond. La reproduction chez la vache, Cambridge, 1927.

[5] Magkenzie, Marshall et Hammond, Journ. Agr. Sc., 1911 à 1916, 4, 5, et 7.

[6] Orta-Jensen. World's Dairy Congress, Londres, 1928. Proceedings.

[7] Porcher. Compte rendu de l'Académie des Sciences, 1904, 138.

[8] Porcher. Le lait au point de vue colloïdal, Lyon, 1929.

[9] Sanders. Journ. Agr. Sc., 1927-28, 17 et 18.

[10] Woodman et Hammond. Journ, Agr. Sc, 1922, 12.

[11] Woodman et Hammond. Journ. Agr. Sc., 1923, 13.

\section{ÉTUDE DES CHAMPIGNONS TROUVÉS DANS LE LAIT LA CRÈME ET LE BEURRE}

$$
\text { par }
$$

\author{
M. GRIMES \\ Section de Bactériologie laitière
}

et

H. A. CUMMINS et V. C. E. KENNELLY

Section de Botanique

Université de Cork (Etat libre d'Irlande)

(Fin).

Chætomium bostrychoides Zopf [16] (P1. 22, fig. 14).

Cette forme, qui est très variable, fut trouvée une fois des périthéces apparaissant dans les cultures. Les périthéces sont gris acier, ovales, mais peuvent 